\title{
CHEMICAL EVOLUTION AND PRIMORDIAL NUCLEOSYNTHESIS
}

\author{
J.Audouze ${ }^{1,2}$ and E.Vangioni-Flam ${ }^{1}$ \\ 1 Institut d'Astrophysique du CNRS, Paris-France \\ 2 Laboratoire René Bernas, Orsay-France
}

\begin{abstract}
This contribution starts with a very brief updated report on our present knowledge concerning the primordial abundances of the lightest elements $\left(\mathrm{D},{ }^{3} \mathrm{He},{ }^{4} \mathrm{He}\right.$ and $\left.{ }^{7} \mathrm{Li}\right)$. From this information it is claimed here that specific models of chemical evolution able to account for a thorough destruction of $\mathrm{D}$ during the galactic history should be involved to reconcile the ratios : $\eta=\eta_{B} / \eta_{\gamma}$ (baryonic density relative to background photon density) predicted respectively from the primordial ${ }^{4} \mathrm{He}(\mathrm{Y})$ abundance and from the primordial $\mathrm{D}$ (and ${ }^{3} \mathrm{He}$ ) abundance. Among different possibilities, it is shown that galactic evolution models implying variations of the role of star formation (SFR) are more successful for that goal than models implying bimodal star formation.From this analysis it is also argued that contrary to a common belief, a large $\mathrm{D}$ destruction rate over the galactic history implies very specific conditions concerning the chemical evolution of our galaxy.
\end{abstract}

\section{INTRODUCTION}

The goal of this contribution is first to advocate that the primordial abundances of the lightest elements $\left(\mathrm{D},{ }^{3} \mathrm{He},{ }^{4} \mathrm{He}\right.$ and $\left.{ }^{7} \mathrm{Li}\right)$ which are presently available lead to somewhat discordant predictions regarding the baryonic density of the Universe when the simplest standard assumptions are adopted for both the models of early nucleosynthesis and of the chemical evolution of our Galaxy. In order to reconcile such predictions in the frame of the simple "canonical" Big Bang model of nucleosynthesis, we felt compelled (see eg Delbourgo-Salvador etal,1985 and Vangioni-Flam and Audouze,1987(VFA)) to propose specific models of galactic evolution leading to a thorough D destruction during the history of our Galaxy. The second goal of this paper is to present a short account of a promising hypothesis : the model of galactic evolution when the rate of star formation (SFR) is supposed to vary with time and which is analysed in detail in VFA. After a very brief review of the primordial abundances of $\mathrm{D},{ }^{3} \mathrm{He},{ }^{4} \mathrm{He}$ and ${ }^{7} \mathrm{Li}$ (section 2 ), 
the problem concerning the discordant baryonic densities deduced from the ${ }^{4} \mathrm{He}$ abundance $(\mathrm{Y})$ on one hand and from $\mathrm{D}$ and ${ }^{3} \mathrm{He}$ on the other hand, is presented in section 3. The specific models of the galactic evolution of $D$ are presented in section 4 and the conclusions of this analysis in section 5 .

\section{THE PRIMORDIAL ABUNDANCES OF THE LIGHTEST ELEMENTS}

$$
\left(\mathrm{D},{ }^{3} \mathrm{He},{ }^{4} \mathrm{He} \text { AND }{ }^{7} \mathrm{Li}\right)
$$

Given the cosmological implications of the primordial abundances of the lightest elements $\left(\mathrm{D},{ }^{3} \mathrm{He},{ }^{4} \mathrm{He}\right.$ and $\left.{ }^{7} \mathrm{Li}\right)$, they are reviewed in many recent papers, see eg Boesgaard and Steigman,1985 and Audouze,1987. An update of the present determination of these abundances is given in some detail in Audouze and VangioniFlam, 1988 and in Audouze, Spite and Spite, 1988. Table 1 represents the summary of their review (see also Beckman and Pagel, this volume for further details.)

TABLE 1

Primordial Abundances of $\mathrm{D},{ }^{3} \mathrm{He},{ }^{4} \mathrm{He}$ and ${ }^{7} \mathrm{Li}$

\begin{tabular}{|c|c|c|c|c|}
\hline Element & Primordial & $\begin{array}{c}\text { Old and low } Z \\
\text { Objects }\end{array}$ & Solar System & $\begin{array}{c}\text { Present Inter- } \\
\text { stellar medium }\end{array}$ \\
\hline $\begin{array}{c}\text { (Age, } \\
\text { Gyr })\end{array}$ & 212 & $10-12$ & $4-6$ & 0 \\
\hline$D$ & $310^{-5}-10^{-4}$ & $(2-8) 10^{-5}$ & $(2 \pm 1) 10^{-5}$ & $(1 \pm 0.3) 10^{-5}$ \\
\hline${ }^{3} \mathrm{He}$ & $310^{-5}-10^{-4}$ & & $(2 \pm 1) 10^{-5}$ & $10^{-5}-1.510^{-4}$ \\
\hline $\mathrm{D}^{3} \mathrm{He}$ & $610^{-5}-210^{-4}$ & & $(2-4) 10^{-5}$ & $210^{-5}-1.610^{-4}$ \\
\hline${ }^{4} \mathrm{He}(\mathrm{Y})$ & $0.24 \pm 0.01$ & $(0.24 \pm 0.01)+\mathrm{AZ}$ & $0.24-0.28$ & $0.24-0.30$ \\
\hline${ }^{7} \mathrm{Li}$ & $\begin{array}{c}(1 \pm 0.3) 10^{-10}\left(8^{2}\right) \\
(2-9) 10^{-10}(\mathrm{DH})\end{array}$ & $810^{-11}-2.510^{-1}$ & $10^{-9}$ & $10^{-9}$ \\
\hline
\end{tabular}




\section{SOME DISCORDANCES IN THE "CANONICAL MODEL OF EARLY NUCLEOSYNTHESIS"}

The remarkable agreement between the primordial abundances of the lightest elements and those computed in the frame of the standard ( simplest) models of Big Bang, (see eg Boesgaard and Steigman,1985) is rightly considered as one of the major successes of this cosmological model. As shown especially in the review, the primordial nucleosynthesis leads to two most exciting constraints (i) on the overall baryonic density which is such that $\Omega_{B} \sim 0.1$ (where $\Omega_{B}$ is the baryonic cosmological parameter) ; this means that the baryonic density represents only $\sim 10 \%$ of the critical density above which the Universe would be closed. In the sequel we will use either $\Omega_{B}$ or $\eta_{B}=\eta_{B} / \eta_{\gamma}$ where $\eta_{B}$ and $\eta_{\gamma}$ are respectively the baryon and the background photon densities. $\Omega_{B}$ is related to $\eta_{B}$ by the relation $\Omega_{B}=3.53 \quad 10^{-3} \quad \eta_{10} \mathrm{~h}^{-2}(\mathrm{~T} / 2.7)^{3}$ where $\eta_{10}=10^{10} \eta_{B} ; \mathrm{h}=\left(\mathrm{H}_{o} / 100\right)$ where $\mathrm{H}_{o}$ is the Hubble constant expressed in $\mathrm{km} \mathrm{s}^{-1} \mathrm{Mpc}^{-1}$ and $\mathrm{T}$ is the temperature of the background radiation; (ii) on the maximum number of neutrino (lepton) families which is about 3 to 4 (remember that three lepton families have already been discovered). This implies that there is no much room for further discoveries of new lepton flavours in the frame of this standard model.

Given the importance of such conclusions, they should be scrutinized very precisely. As discussed at some length eg in Audouze 1987 we claim that the primordial abundance of $\left(\mathrm{D}+{ }^{3} \mathrm{He}\right)$ used in Boesgaard and Steigman (1985) and the primordial abundance of ${ }^{4} \mathrm{He}\left(\mathrm{Y}_{p}\right)$ are only consistent for an extremely narrow range of $\eta_{10}\left(\eta_{10}=3.2 \pm 0.2\right)$. This conclusion has also been reached by Pagel (1986). This narrow range corresponds to $\Omega_{b}=0.01$ to 0.04 : the width of that range is only due to the fact that the "battle" between the $\mathrm{H}_{o}=50 \mathrm{~km} \mathrm{~s}^{-1} \mathrm{Mpc}^{-1}$ proponents and those who claim that $\mathrm{H}_{0}=100 \mathrm{~km} \mathrm{~s}^{-1} \mathrm{Mpc}^{-1}$ is not yet ended. One should note also that this very restricted agreement may disappear if $Y_{p}$ is found to be as low as 0. 23 (Beckman and Pagel, this conference).

In the analysis of Yang et al 1984, on which the Boesgaard and Steigman (1985) review is based, the primordial $\left(\mathrm{D}+{ }^{3} \mathrm{He} / \mathrm{H}\right) \leq 10^{-4}$ value is determined by assuming that the chemical evolution of our Galaxy is properly described by the simplest models such as those of Audouze and Tinsley (1974). In such models the overall $\mathrm{D}$ destruction is limited in such a way that $\mathrm{D}_{\text {primordial }} / \mathrm{D}_{\text {present }} \leq 3-4$.

This is why we felt compelled to examine the possibility of considering models of chemical evolution leading to higher primordial values of $\mathbf{D}$ consistent with lower values of ${ }^{4} \mathrm{He}$.

\section{SPECIFIC MODELS OF GALACTIC EVOLUTION LEADING TO A THOROUGH DESTRUCTION OF D}

Up to a quite recent past, we (Delbourgo-Salvador et al 1985) have proposed two types of models able to destroy thoroughly $\mathrm{D}$ during the galactic history that we 
will not review again here : they are (i) an inflow of processed (D free) material inside the solar vicinity and (ii) the ejection of $D$ free material by stellar winds during the premain sequence. Following our analysis (VFA), we will consider here two specific models related to recent ideas put forward eg by Larson 1986, Wyse and Silk (WS) 1987, Audouze et al 1987, concerning both varying rate of star formation (SFR) and bimodal SFR. The first model hereafter referred to as model II has been designed to study the effect of a large SFR at the beginning of the galactic history. Model I is the standard one (Audouze 1987) with SFR $\psi(\mathrm{t})$ proportional to the gas density $\sigma$ such that $\nu$ the constant of proportionality is $\nu=0.3$ $\mathrm{Gyr}^{-1}$ and a monomodal IMF $\Phi(\mathrm{m}) \alpha m^{(-x+1)}$ with $\mathrm{x}=2$ and $0.4 \leq \mathrm{m} / \mathrm{Mo} \leq 100$. In model II, the SFR parameter $\Psi(\mathrm{t})$ has been modified on the following way : $\Psi_{2}(\mathrm{t})=\nu_{2}$ for $\mathrm{t}<\tau \mathrm{Gyr}$ and $\Psi_{1}(\mathrm{t})=\nu_{1} \sigma$ for $\mathrm{t}>\tau(\mathrm{Gyr})$. In this model we have conserved the classical IMF with $\mathrm{x}=2$ for the whole stellar mass spectrum 0.4-100Mo. The parameters $\nu_{2}$ and $\tau$ are such that $0.4 \leq \nu_{1} \leq 2$ and $2 \geq \tau \geq 0.5 \mathrm{Gyr}$ while $\nu_{1}=0.3$

Model III has been designed to study the effect of bimodal SFR. It follows the WS prescriptions: the number of stars formed in the mass interval $d m$ during a time $d t$ is :

$$
d^{2} n(m, t)=\left[\Phi_{1}(m) / \Psi_{1}(t)+\Phi_{2}(t) \Psi_{2}(t)\right] d m d t
$$

The first term at the right hand side of (1) represents a constant mode of star formation from 0.4 to $100 \mathrm{Mo}$ such as $\Psi_{1}(\mathrm{t})=\nu_{1}$, the second term represents a massive mass mode from the lower mass limit $m_{L 2}(1$ to $2 \mathrm{Mo})$ to $100 \mathrm{Mo}$ such that $\Psi_{2}(\mathrm{t})=\nu_{2} \cdot e^{-t / \tau}$. The corresponding IMF has the same slope $\mathrm{x}=2$ but different lower mass limits. The parameters $\nu_{1}, \nu_{2}$ and $\tau$ are $\nu_{1}=0.06, \nu_{2}=0.3$ and $\tau=2 \mathrm{Gyr}$. Finally for reasons which are discussed below, we have also considered model IV which is similar to model III but where the first constant SFR term in the right hand side of equation (1) is replaced by $\Psi_{1} t_{1}=\nu_{1} \sigma$ i.e. decreasing proportionally to the gas density.

Table 2 provides an account of the galactic evolution corresponding to these three different models, Figures 1, 2 and 3 display respectively the evolution of the normalized gas density $(\sigma)$, the overall metallicity $(z)$ and the $D$ mass abundance $\left(X_{D}\right)$.

To sum up the discussion of these models which can be found in VFA the following points should be stressed :

a) Model II (with varying SFR) is the model which is the best suited to account (at the same time for the evolution of $\sigma, \mathrm{Z}$ and a large $X_{D}$ destruction. This is because gas processing is due to stars of any mass. Moreover as shown by Andreani et al 1988 that type of evolution model is also able to reproduce most satisfactorily the evolution of heavier elements $(\mathrm{N}, \mathrm{O}, \mathrm{Fe}$, the s process and the $r$ process elements). The only price to pay is that this model may not be satisfactory enough to reproduce properly the overall present luminosity function 
Table 2

\begin{tabular}{|c|c|c|c|c|c|c|}
\hline & $\begin{array}{l}\text { Primordial } \\
\text { Phase } \\
t \simeq 100 \mathrm{~s}\end{array}$ & $T_{0}=1$ & $2.5 \mathrm{Gyr}$ & & & \\
\hline & $\begin{array}{l}\text { Models } \\
\text { IId, IVa,b,c }\end{array}$ & $\begin{array}{l}\text { Observational } \\
\text { constraints }\end{array}$ & Model IId & Model IVa & Model IVb & Model IV \\
\hline$\sigma=\frac{M_{\text {gate }}}{M_{\text {todel }}}$ & 1 & 0.05 to 0.1 & 0.05 & 0.06 & 0.05 & 0.06 \\
\hline H & 0.76 & $\sim 0.70$ & 0.69 & 0.6 & 0.63 & 0.62 \\
\hline D & $10^{-4}$ & $510^{-6} \leq \mathrm{D} \leq 1.510^{-5}$ & $0.1510^{-4}$ & $0.110^{-4}$ & $0.110^{-4}$ & $0.110^{-4}$ \\
\hline$\frac{D_{\text {prim }}}{D_{\text {preo }}}$ & 1 & & $\sim 7$ & $\sim 10$ & $\sim 10$ & $\sim 10$ \\
\hline${ }^{3} \mathrm{He}$ & $510^{-5}$ & $(2-7) 10^{-5}$ & $6.610^{-5}$ & $310^{-5}$ & $3.910^{-5}$ & $3.510^{-5}$ \\
\hline${ }^{4} \mathrm{He}$ & 0.24 & $\sim 0.28$ & 0.29 & 0.33 & 0.33 & 0.33 \\
\hline z & 0 & $\sim 0.02$ & 0.02 & 0.06 & 0.04 & 0.05 \\
\hline
\end{tabular}

Resulting values of two models: Model IId, where $\psi_{1}(t)=\nu_{1} \sigma, \nu_{1}=0.3, \mathrm{t} \geq 1 \mathrm{Gyr}, \psi_{2}(t)=\nu_{2}$, $\nu_{2}=0.8, \mathrm{t} \leq 1 \mathrm{Gyr}$, Model IVa, where $\psi_{1}(t)=\nu_{1} \sigma, \nu_{1}=0.3, \psi_{2}(t)=\nu_{2} e^{-t / 2}, \nu_{2}=0.3$, the IMF parameters are $x=2$ and $m_{L 2}=2 \mathrm{M}_{\odot}$. Model IVb has the same parameters than Model IVa but with an IMF slope $x=2.2$. Model IVc has the same parameters than Model IVa but with a low mass limit for the massive mode $m_{L 2}=1.5 \mathrm{M}_{\odot}$.

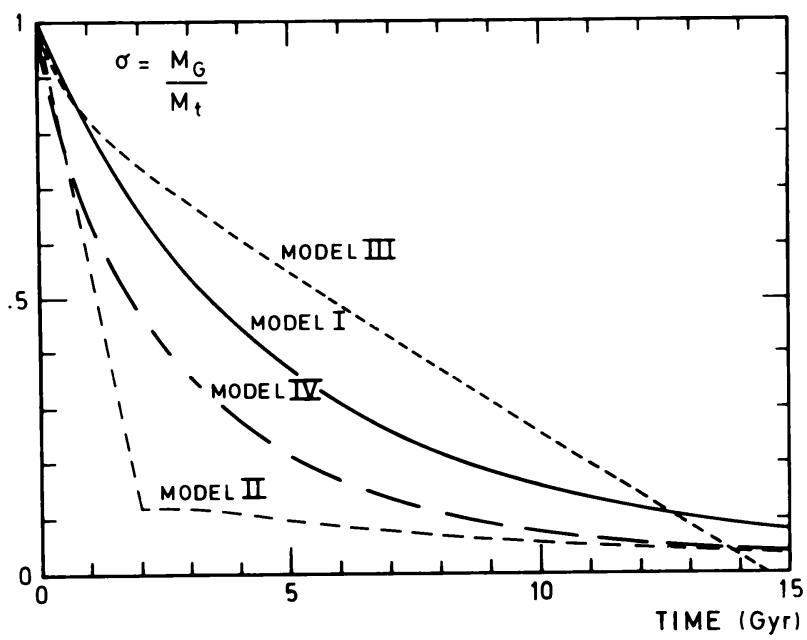

Figure 1. The evolution of normalized surface gas density $\left(\sigma=\mathrm{M}_{\text {gae }} / \mathrm{M}_{\text {total }}\right)$ is presented for 4 models : - Model I, standard rate of star formation : $\psi(\mathrm{t})=0.3 \sigma \phi(\mathrm{m}) \propto \mathrm{m}^{-3} \quad 0.4 \leq \mathrm{m} / \mathrm{M}_{\odot} \leq$ 100 - Model II, two regimes in the rate of star formation : $\psi_{2}(\mathrm{t})=0.5$ if $t \leq 2 \mathrm{Gyr} \psi_{1}(\mathrm{t})=0.3 \sigma$ if $t \geq 2 \mathrm{Gyr} \phi(m) \propto m^{-3} \quad 0.4 \leq m / M_{\odot} \leq 100$ - Model III, bimodal rate of star formation $\psi_{2}(t)=0.3 e^{-t / 2} \phi_{2} \propto m^{-3} \quad 2 \leq m / M_{\odot} \leq 100 \psi_{1}(t)=0.06 \quad \phi_{1} \propto m^{-3} \quad 0.4 \leq m / M_{\odot} \leq 100$ - Model IV, $\psi_{2}(t)=0.3 e^{-t / 2} \phi_{2} \propto m^{-3.2} \quad 2<m / M_{\odot}<100 \psi_{1}(t)=0.3 \sigma \quad \phi_{1} \propto m^{-3.2} \quad 0.4<$ $m / M_{\odot}<100$ 
608

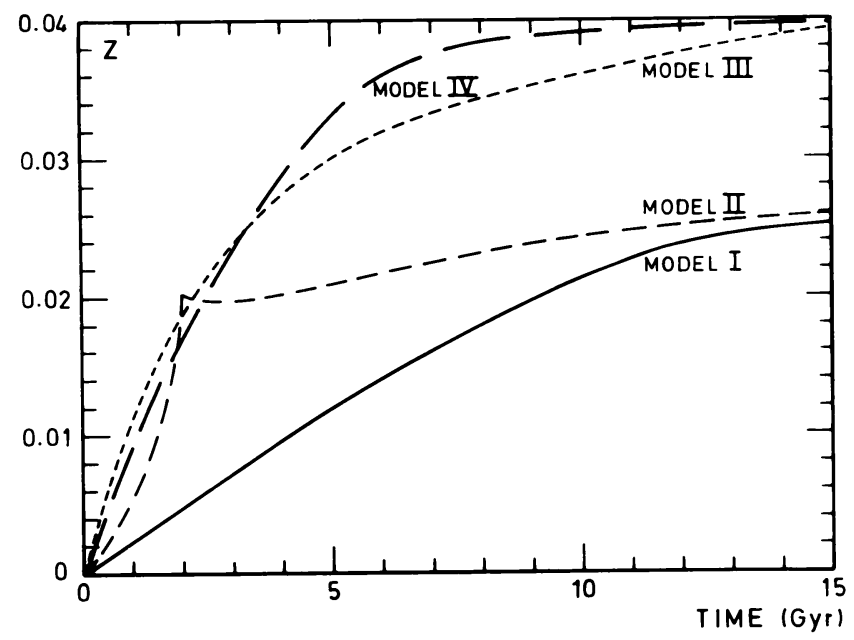

Figure 2. The evolution of metallicity $Z$ for the same models as in figure 1 .

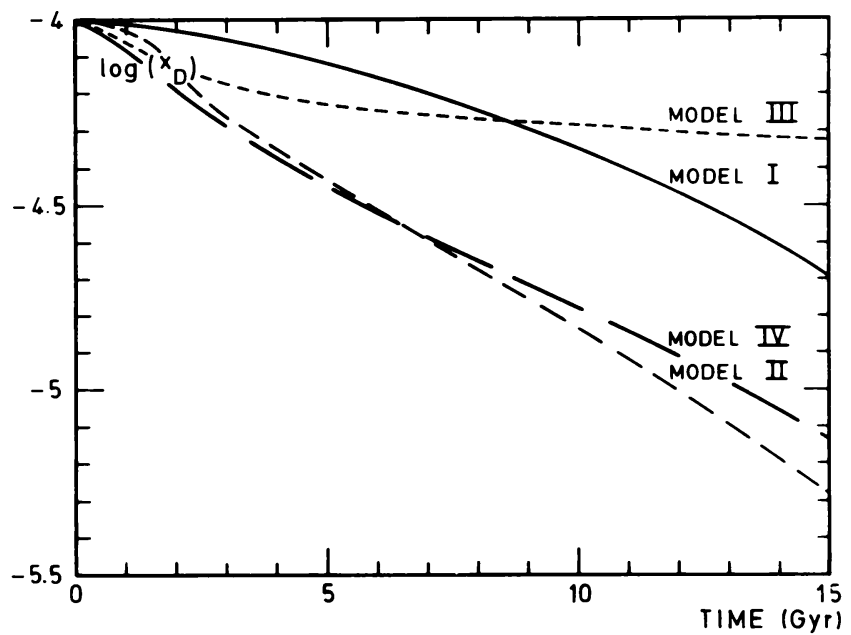

Figure 3. The evolution of deuterium (in mass) for the same models as in figures 1 and 2. 
of stars. We (VFA) have proposed a slight modification of the parameters of this model $\left(\nu_{2}=0.8\right.$ and $\left.\tau=1\right)$ which satisfy the Scalo (1986) SFR limits imposed by the stellar luminosity function. But this modification decreases the $\mathrm{D}$ destruction $\left(D_{\text {primordial }} /\right.$ Dpresent $=7$ instead of 10-13).

b) Model III is indeed well suited to reproduce satisfactorily the evolution of the luminosity function (WS) but leads to an overmetallicity (too high Z) and a much too low D destruction. As discussed thoroughly in VFA, an only formation of massive stars at the very beginning of the galactic evolution does not imply necessarily a large D destruction while low mass stars evolve slowly and eject D free material and few metals (since $D$ is destroyed in any stellar environment), by contrast high mass stars evolve quickly but destroy $D$ at the expense of rejecting metal rich stars. This is why in evolution models like model III SFR has to be strongly limited in order to avoid a too large overproduction of metals.

c) Since type II models are successful in accounting for the D destruction but not as good regarding the present luminosity stellar function while it is reverse for type III models, we have considered model IV which is an hybrid of models II and III : this model satisfies quite properly the $\mathrm{D}$ and stellar luminosity requirements but still leads to a metallicity (too high by a factor 2) and to an ${ }^{4} \mathrm{He}(\mathrm{Y})$ abundance of 0.33 which is also much too high.

\section{SUMMARY AND CONCLUSIONS}

The chemical evolution of the lightest elements produced by the Big Bang nucleosynthesis is indeed not the only exciting problem which one has to adress itself now : the early nucleosynthesis possibly affected by the quark- hadron phase transition is presently inspiring almost all the specialists involved in this field (see eg Applegate et al,1987, Alcock et al,1987, Audouze et al,1988 and Reeves,1988) . Nevertheless this contribution devoted to these evolutionary aspects gives us an opportunity to draw the attention of the interested reader on the complementarity which exists between the early nucleosynthesis (and therefore its implications on cosmology and particle physics). As discussed eg by Boesgaard and Steigman 1985, Audouze 1987, and Reeves et al 1988 standard Big Bang nucleosynthesis leading to an upper limit of $\Omega_{B} \leq 0.1$ seems still to be the most secure hypothesis. We claim here that in order to alleviate any difficulty which could arise from some discordance between the predictions on $\Omega_{B}$ coming from ${ }^{4} \mathrm{He}$ on one hand and $\left(\mathrm{D}+{ }^{3} \mathrm{He}\right)$ on the other hand, $\mathrm{D}$ has to be thoroughly destroyed during the galactic history. In order to achieve that goal some specific evolution models are required the most promising being those assuming time varying SFR (although some "fine tuning" are required in order to avoid any difficulty which could arise from the stellar luminosity function). Such time varying SFR (models may also be found useful in other contexts such as the evolution of heavier elements (Andreani et al 
1988). Evolution models implying a bimodal IMF such as those of WS, are not successful in that respect since they are unable to lead to a thorough $D$ destruction during the galactic history. Finally, one should note that only very specific evolution models are able to lead to such a D galactic destruction. Although this element is extremely sensitive to any extraction process its overall destruction is contingent to conditions on evolutionary parameters such as SFR. The apparent simplicity of the standard Big Bang nucleosynthesis and its quite remarkable consequences on the baryonic density of the Universe and the maximum number of families of relativistic particles (such as neutrinos) may require some somewhat contrived galactic evolution models.

\section{ACKNOWLEDGEMENTS}

This contribution has been efficiently typed by M.C.Pantalacci. The research reported here has been supported in part by PICS 18.

\section{REFERENCES}

Alcock,C.R., Fuller,G.M. and Mathews,G.J., 1987, Ap.J. (in press)

Andreani,P., Vangioni-Flam,E. and Audouze,J., 1988, Ap.J. (submitted)

Applegate,J.H., Hogan,C.T. and Scherrer,R.J., 1987, Phys.Rev. D 35,1151

Audouze,J., 1987, in Observational Cosmology, eds A.Hewitt et al, ReidelDordrecht, p.89

Audouze,J., Delbourgo-Salvador,P. and Salati,P.,1988, eds J.Audouze and F. Melchiorri, Varenna (in press)

Audouze,J., Delbourgo-Salvador,P. and Vangioni-Flam,E., 1987, in Advances in

Nuclear Astrophysics, eds E.Vangioni-Flam et al, Editions Frontières p.47

Audouze,J., Spite,F. and Spite M., 1988, Phys. Reports (in preparation)

Audouze,J. and Tinsley,B.M., 1974, Ap.J. 192, 487

Audouze,J. and Vangioni-Flam,E., 1988, in Varenna proceedings, eds J.Audouze and F.Melchiorri (in press)

Boesgaard,A.M. and Steigman,G., 1985, Ann. Rev. Astron. Astroph., 23,319

Cameron,A.G.W., 1982, Essays in Nuclear Astrophysics, eds C.A.Barnes et al, Cambridge Univ. Press, p.23

Delbourgo-Salvador,P., Gry,C., Malinie,G. and Audouze,J., 1985, Astron. Astroph., 150, 53

Larson,R.B., 1986, MNRAS, 218, 409

Meyer,JP., 1985, Ap.J. suppl., 41, 513

Peimbert,M., 1986, in Star forming regions, eds M.Peimbert and J.Jugaku, ReidelDordrecht

Reeves,H., 1988," Confrontation between observations and theories in Cosmology", in Varenna proceedings, eds J.Audouze and F.Melchiorri,(in press) 
Reeves,H., Delbourgo-Salvador,P., Salati,P. and Audouze,J., 1988, Phys. Rev. $\underline{\text { }}$, (submitted)

Rood,R.T., Bania, T.M., Wilson,T.L., 1984, Ap.J., 280, 629

Sanders,D.B., Solomon, P.M., Scoville, N.Z., 1984, Astron. Astroph., 276, 182

Scalo, J.M., 1986, Fundam. Cosm. Phys., 11, 1

Vangioni-Flam,E. and Audouze,J., 1987, Astron. Astroph., (in press)

Vidal-Madjar,A.,1987, in Space Astronomy and Solar System Exploration, ESA SP 268, p.73

Wyse,R. and Silk,J., 1987, Ap.J. Letters, $\underline{313}$, L11

\section{DISCUSSION}

Gustafsson : You honted at the possibility to use heavier element abundances for proving or disproving the bi-modal star-formation hypothesis. What particular suggestions would you make?

Audouze : Our current analysis (P.Andreani, E.Vangioni-Flam and myself) concerning the chemical evolution of heavier elements shows clearly that models with varying rate of star formation are more successful than those assuming a bimodal initial mass function.

Beckman : It appears that we are left with the choice of fine-tuning the galaxy or fine -tuning the Universe!

Audouze : I do not agree entirely with this statement. It seems more satisfactory to find out a general solution for the early universe to the expense of fine- tuning the galaxy. But for me it is still an open question.

\section{Boesgaard :}

Your models can predict the $\mathrm{Li}$ astration also. How you done that?

Audouze :

We should indeed determine as precisely as possible the chemical evolution of ${ }^{7} \mathrm{Li}$ by taking into account both destruction and formation processes. We plan to study this problem in a very near future. However we know already especially from the work I did with Beatrice Tinsley some years ago that ${ }^{7} \mathrm{Li}$ will be much less affected than $D$ by destruction processes. 